\title{
Dynamical decoherence of the light induced interlayer coupling in $\mathrm{YBa}_{2} \mathrm{Cu}_{3} \mathrm{O}_{6+\delta}$
}

\author{
C. R. Hunt,,${ }^{1,2}$ D. Nicoletti, ${ }^{1}$ S. Kaiser,,${ }^{1,3,}{ }^{*}$ D. Pröpper, ${ }^{3}$ T. Loew,${ }^{3}$ J. Porras,${ }^{3}$ B. Keimer, ${ }^{3}$ and A. Cavalleri ${ }^{1,4}$ \\ ${ }^{1}$ Max Planck Institute for the Structure and Dynamics of Matter, Hamburg, 22761, Germany \\ ${ }^{2}$ Department of Physics, University of California Berkeley, Berkeley, California 94720, USA \\ ${ }^{3}$ Max Planck Institute for Solid State Research, Stuttgart, 70569, Germany \\ ${ }^{4}$ Department of Physics, Oxford University, Clarendon Laboratory, Oxford OX1 3PU, United Kingdom
}

(Received 3 August 2016; published 12 December 2016)

\begin{abstract}
Optical excitation of apical oxygen vibrations in $\mathrm{YBa}_{2} \mathrm{Cu}_{3} \mathrm{O}_{6+\delta}$ has been shown to enhance its $c$ axis superconducting-phase rigidity, as evidenced by a transient blueshift of the equilibrium interbilayer Josephson plasma resonance. Surprisingly, a transient $c$ axis plasma mode could also be induced above $T_{c}$ by the same apical oxygen excitation, suggesting light activated superfluid tunneling throughout the pseudogap phase of $\mathrm{YBa}_{2} \mathrm{Cu}_{3} \mathrm{O}_{6+\delta}$. However, despite the similarities between the transient plasma mode above $T_{c}$ and the equilibrium Josephson plasmon, alternative explanations involving high-mobility quasiparticle transport should be considered. Here, we report an extensive study of the relaxation of the light induced plasmon into the equilibrium incoherent phase. These new experiments allow for a critical assessment of the nature of this mode. We determine that the transient plasma relaxes through a collapse of its coherence length rather than its carrier (or superfluid) density. These observations are not easily reconciled with quasiparticle interlayer transport and rather support transient superfluid tunneling as the origin of the light induced interlayer coupling in $\mathrm{YBa}_{2} \mathrm{Cu}_{3} \mathrm{O}_{6+\delta}$.
\end{abstract}

DOI: 10.1103/PhysRevB.94.224303

\section{INTRODUCTION}

Underdoped cuprates retain some of the properties associated with superconductivity above the transition temperature $T_{c}$ [1-7]. For instance, unlike conventional superconductors, in which the transition to the normal state is governed by a reduction in condensate density, superconductivity in cuprates is also weakened by fluctuations of the order parameter phase. These fluctuations set in below $T_{c}[8-10]$ and persist above the transition temperature [11-14]. Competing orders-such as the charge and spin stripes found in lanthanides [15-19] and other charge density wave states observed across a wide range of cuprates [20-23] - may disrupt superconductivity through order parameter modulation [24-27].

Targeted ultrafast light excitation has been recently shown to transiently restore superconducting-like properties above equilibrium $T_{c}$. This is most directly evidenced by the appearance of plasma modes reminiscent of intrinsic Josephson coupling between the $\mathrm{CuO}_{2}$ layers [28-36]. In a first set of experiments, light induced interlayer coupling was observed in single-layer lanthanide $\mathrm{La}_{1.8-x} \mathrm{Eu}_{0.2} \mathrm{Sr}_{x} \mathrm{CuO}_{4}$ after excitation resonant to a $\mathrm{Cu}-\mathrm{O}$ stretching mode [37]. Because this effect correlates with the static charge order [38], and because the same excitation was also shown to disrupt stripe order in the related compound $\mathrm{La}_{0.1875} \mathrm{Ba}_{0.125} \mathrm{CuO}_{4}$ [39], the transient appearance of superconductivity can probably be explained in 1/8-doped single-layer cuprates as a result of competing order melting.

More puzzling has been the response of underdoped $\mathrm{YBa}_{2} \mathrm{Cu}_{3} \mathrm{O}_{6+\delta}[40,41]$, for which excitation of the apical oxygen atoms, sitting above and below the $\mathrm{CuO}_{2}$ planes [see Fig. 1(a)], also promotes a superconducting-like plasma mode. Whereas the effect was correlated with suppression of charge density wave order [42] near $1 / 8$ hole doping $\left(\mathrm{YBa}_{2} \mathrm{Cu}_{3} \mathrm{O}_{6.65}\right)$, measurements at lower dopings

\footnotetext{
*Present address: 4th Physics Institute, University of Stuttgart, Stuttgart, 70550, Germany.
}

( $\mathrm{YBa}_{2} \mathrm{Cu}_{3} \mathrm{O}_{6.5}$ and $\mathrm{YBa}_{2} \mathrm{Cu}_{3} \mathrm{O}_{6.45}$ ) generated light induced coherence at even higher temperatures. Because equilibrium charge order is weak for these doping levels, the results in $\mathrm{YBa}_{2} \mathrm{Cu}_{3} \mathrm{O}_{6+\delta}$ are difficult to reconcile with a competing order melting, and they also raise questions on the nature of the plasma mode itself. Provided that the plasma edge can be uniquely associated with Josephson tunneling also for all underdoped $\mathrm{YBa}_{2} \mathrm{Cu}_{3} \mathrm{O}_{6+\delta}$ samples, these experiments indicate that light induced superconductivity may result from a more general mechanism $[43,44]$.

\section{EXPERIMENT}

Here, we present a comprehensive study of the lightinduced interlayer coupling and especially its relaxation in $\mathrm{YBa}_{2} \mathrm{Cu}_{3} \mathrm{O}_{x}$ (YBCO $x$ ). Five different compounds were studied, consisting of underdoped $x=6.3,6.45,6.5,6.6$, and optimally doped $x=7$, with $T_{c} \simeq 0 \mathrm{~K}, 35 \mathrm{~K}, 50 \mathrm{~K}, 62 \mathrm{~K}$, and $90 \mathrm{~K}$, respectively. The single crystals used in the experiment [40] had typical dimensions of $2 \times 2 \times 1 \mathrm{~mm}^{3}$. Their $T_{c}$ values were determined by direct current (dc) magnetization measurements [see Fig. 1(b)]. YBCO 6.3 was found to be nonsuperconducting down to the lowest measured temperature.

We photoexcited YBCO with 300 fs mid-infrared pulses, polarized perpendicular to the $\mathrm{CuO}_{2}$ planes (along the $c$ axis) and with field strengths up to $\sim 3 \mathrm{MV} / \mathrm{cm}$. These pump pulses were tuned to $15 \mu \mathrm{m}$ wavelength, to resonantly drive a phonon mode of $\mathrm{B}_{1 \mathrm{u}}$ symmetry, involving motion of the apical oxygen atoms [see Fig. 1(a)] [40-43]. The transient optical response was interrogated using delayed single-cycle terahertz $(\mathrm{THz})$ pulses with spectral bandwidth covering the $0.5-2.5 \mathrm{THz}$ range. The experiment was performed in reflection geometry, with the pump beam striking the sample at normal incidence and the $\mathrm{THz}$ probe at $30^{\circ}$. The reflected probe field in the absence of excitation, $E(t)$, and the pump-induced changes to the field at each time delay $\tau, \Delta E(t, \tau)$, were measured via electro-optic sampling and then independently Fourier transformed to retrieve the complex response functions of the photostimulated material (see Supplemental Material S1 [45]). 
(a)

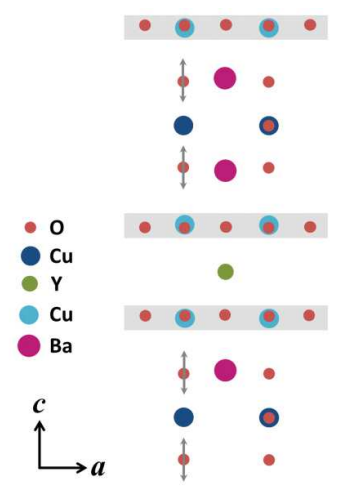

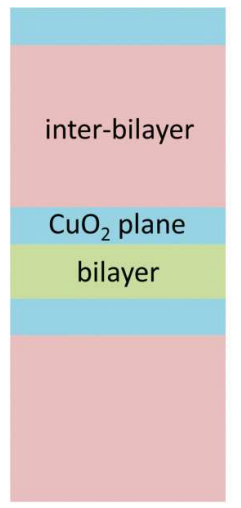

(c)
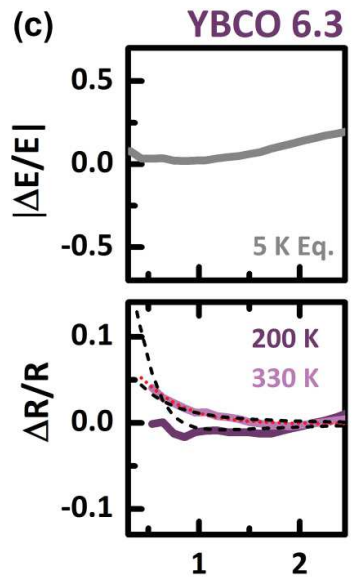

$\omega(\mathrm{THz})$
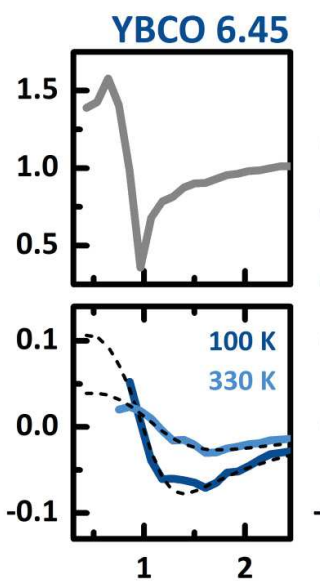

$\omega(\mathrm{THz})$ (b)

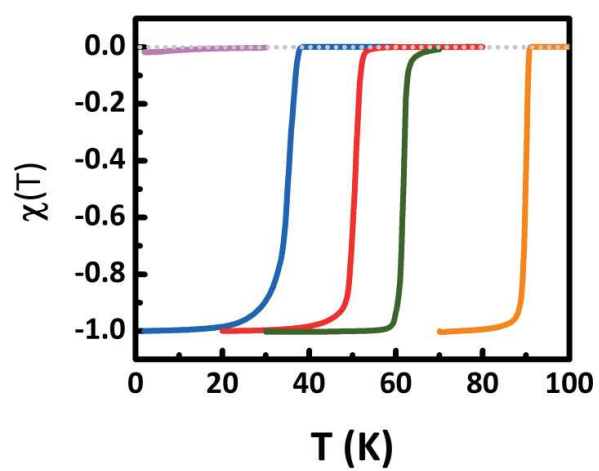

YBCO 6.5
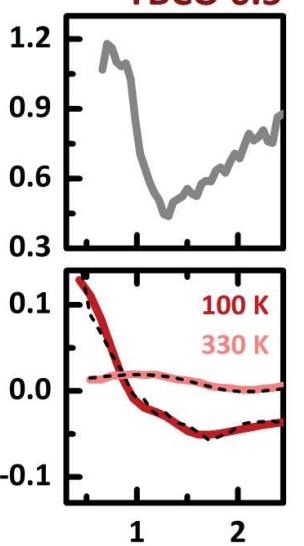

$\omega(\mathrm{THz})$

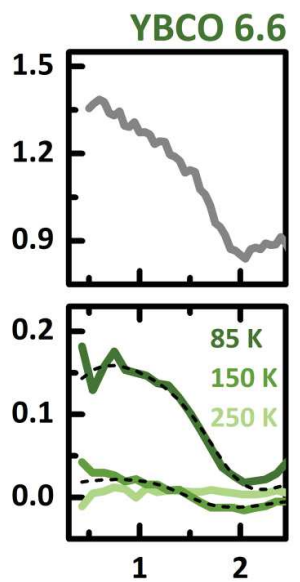

$\omega(\mathrm{THz})$

FIG. 1. Equilibrium superconducting response of YBCO and transient THz reflectivity. (a) Crystal structure of YBCO. Gray arrows indicate the apical oxygen excitation. The unit cell can be divided into two regions, the bilayer units and the interbilayer gaps, separated by $\mathrm{CuO}_{2}$ planes. (b) Equilibrium magnetic susceptibility of the five samples of $\mathrm{YBa}_{2} \mathrm{Cu}_{3} \mathrm{O}_{x}$, with $x=6.3$ (purple), 6.45 (blue), 6.5 (red), 6.6 (green), and 7 (orange). These correspond to hole dopings of $0.05,0.07,0.09,0.12$, and 0.16 , respectively. The $x=6.3$ sample is non-superconducting, while the other samples have transition temperatures of $T_{c}=35 \mathrm{~K}, 51 \mathrm{~K}, 62 \mathrm{~K}$, and $90 \mathrm{~K}$, respectively. (c, top row) Change in the equilibrium $\mathrm{THz}$ reflection coefficient upon cooling below $T_{c}$. Sharp edges are found in the superconducting samples at the Josephson plasma resonance frequency. (c, bottom row) Light induced reflectivity changes measured for different $T>T_{c}$, at $0.8 \mathrm{ps}$ after excitation. Fits with an effective medium model (see main text) are displayed as dashed lines.

The same time-domain setup was also used, in the absence of a pump beam, to determine the THz response of YBCO in its equilibrium superconducting state. The normalized difference $|\Delta \tilde{\mathrm{E}}(\omega) / \tilde{\mathrm{E}}(\omega)|$ between the $c$ axis reflection coefficient at $5 \mathrm{~K}$ and that at $T \gtrsim T_{c}$ was measured for all underdoped samples. This response is dominated by Cooper pair tunneling, as the reflectivity above $T_{c}$ is flat and featureless in this frequency range at all dopings (see Supplemental Material S2 [45]). As shown in the top row of Fig. 1(c), a sharp edge appears for $x=6.45,6.5$, and 6.6 at the Josephson plasma frequency $\omega_{p}$. The edge position blueshifts with increasing doping $x$, and no mode is found for non-superconducting YBCO 6.3.

\section{RESULTS AND DISCUSSION}

\section{A. Light-induced plasma mode}

The pump-induced changes in reflectivity, $\Delta R(\omega, \tau=0.8 \mathrm{ps}) / R(\omega)$, are displayed in the same frequency range in the bottom row of Fig. 1(c) for all dopings at several temperatures above $T_{c}$. These were calculated by taking into account the pump-probe penetration depth mismatch [37,38,40,41], as discussed in Supplemental Material S1 [45]. In analogy with the equilibrium superconducting state, the photo-excited state is characterized by the appearance of a reflectivity edge, the frequency of which blueshifts with increasing doping, tracking the position of the equilibrium Josephson plasma resonance at $T \ll T_{c}$. In YBCO 6.3, only a weak upturn in $\Delta R / R$ was found at low frequencies, suggesting that a mode may be appearing outside of the probed spectral range (see later discussion).

The transient response of the light induced state could be fitted by assuming an effective medium $[40,41]$ consisting of a volume $1-f$ that retains the optical properties of the material at equilibrium (with complex dielectric function $\tilde{\varepsilon}_{b}=\tilde{\varepsilon}_{\text {eq }}$ ), and a volume fraction $f$ with the response of a single plasma mode, described as $\tilde{\varepsilon}_{a}=\tilde{\varepsilon}_{c}-4 \pi \tilde{\sigma}_{p} / i \omega$, where

$$
\tilde{\sigma}_{p}=\frac{1}{4 \pi}\left(\frac{\omega_{p}^{2}}{\Gamma-i \omega}\right) \text {. }
$$



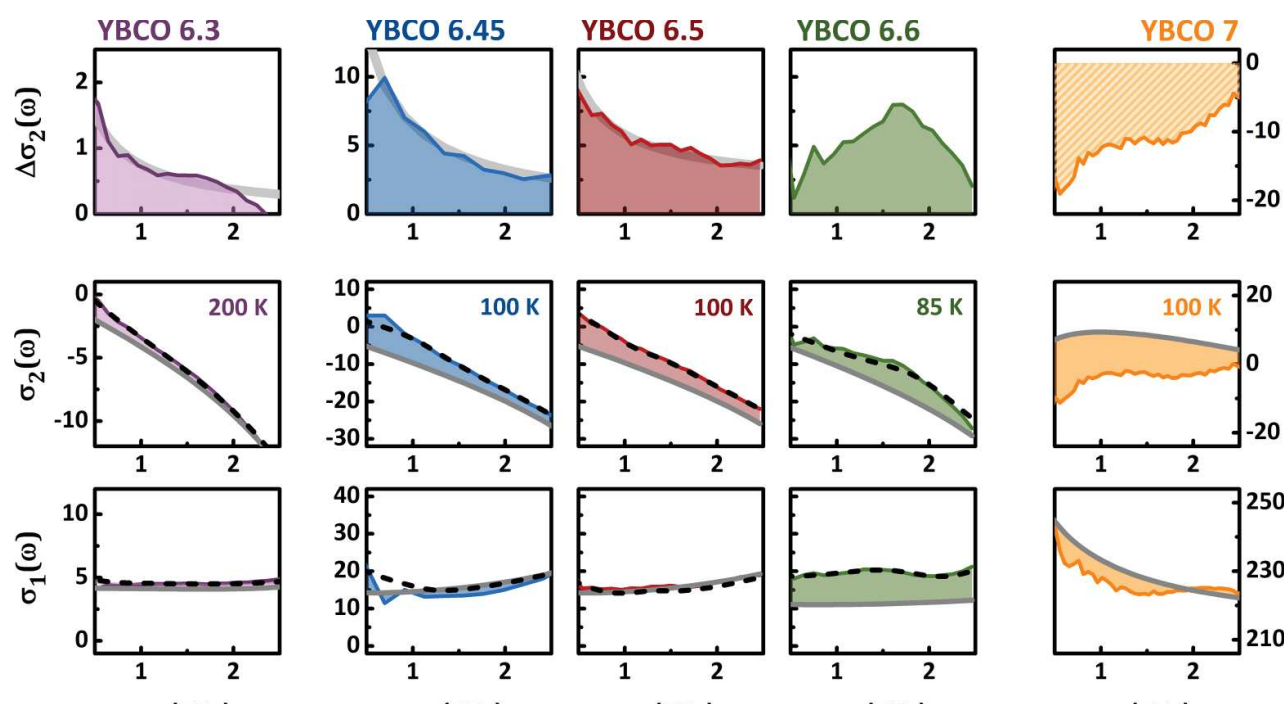

$\omega(\mathrm{THz})$

$\omega(\mathrm{THz})$

$\omega(\mathrm{THz})$

$\omega(\mathrm{THz})$

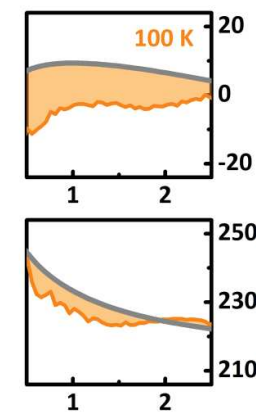

$\omega(\mathrm{THz})$

FIG. 2. Transient complex optical conductivity. (Top row) Pump-induced changes to the imaginary conductivity $\Delta \sigma_{2}(\omega)=$ $\sigma_{2}(\omega, \tau=0.8 \mathrm{ps})-\sigma_{2, \mathrm{eq}}(\omega)$. Gray lines indicate $1 / \omega$ fits. A quasiparticle contribution reshapes $\Delta \sigma_{2}(\omega)$ in YBCO 6.6. (Middle-bottom rows) Corresponding imaginary (middle row) and real (bottom row) parts of the optical conductivity at equilibrium (gray) and at $\tau=0.8 \mathrm{ps}$ (colored by doping), expressed in units of $\Omega^{-1} \mathrm{~cm}^{-1}$. Shaded regions highlight the pump-induced changes. Fits with an effective medium model are shown as dashed lines.

The $\Gamma$ term encompasses all scattering and decoherence processes affecting the transient plasma mode. The effective response $\tilde{\varepsilon}_{E}$ is given by the Bruggeman equation,

$$
f\left(\frac{\tilde{\varepsilon}_{a}-\tilde{\varepsilon}_{E}}{\tilde{\varepsilon}_{a}+2 \tilde{\varepsilon}_{E}}\right)+(1-f)\left(\frac{\tilde{\varepsilon}_{b}-\tilde{\varepsilon}_{E}}{\tilde{\varepsilon}_{b}+2 \tilde{\varepsilon}_{E}}\right)=0 .
$$

Effective medium fits to the $\mathrm{THz}$ reflectivity are shown as dashed lines in Fig. 1(c).

Associated with the appearance of a plasma mode in reflectivity, there is an increase in imaginary conductivity, $\sigma_{2}(\omega)$, which for dissipationless transport should diverge like $1 / \omega$ for $\omega \rightarrow 0$. In Fig. 2, we report the real (middle row) and imaginary (bottom row) parts of the optical conductivity measured at equilibrium and at $\tau=0.8$ ps after excitation. The pump-induced changes to the imaginary conductivity, $\Delta \sigma_{2}(\omega)=\sigma_{2}(\omega)-\sigma_{2, \text { eq }}(\omega)$, are also shown in the top row of the same figure. At all dopings $x \leqslant 6.5, \sigma_{2}(\omega)$ exhibits an enhancement toward low frequencies, while $\sigma_{1}(\omega)$ shows little change and remains gapped, as expected for the purely inductive response of a perfect conductor. In YBCO 6.6, where the hole concentration is higher, a small, frequencyindependent increase in $\sigma_{1}(\omega)$ is observed, which we attribute to incoherent quasiparticle excitation [46]. This contribution also reshapes $\sigma_{2}(\omega)$. Dashed lines represent here the same effective medium fits applied in Fig. 1(c).

While signatures of light induced coherence were already reported for YBCO 6.45-6.6 [40], here we show similar evidence also in YBCO 6.3, which is never a superconductor at equilibrium. As in the other compounds, this response is identified through a positive, $1 / \omega$-type contribution to $\sigma_{2}(\omega)$, following Eq. 1 in the limit $\Gamma \rightarrow 0$. The associated reflectivity edge is not clearly visible, as it remains below our $\mathrm{THz}$ probe spectral window (see Supplemental Material S3 [45] for extended data sets).
Optimally doped YBCO 7 was the only sample for which no light induced increase in $\sigma_{2}(\omega)$ could be found at any temperature (right column of Fig. 2). The pump-induced decrease in both $\sigma_{1}(\omega)$ and $\sigma_{2}(\omega)$ observed in this compound is consistent with quasiparticle heating and supports a scenario in which coherent interlayer transport can only be induced in the pseudogap phase of YBCO. We also note that the $15 \mu \mathrm{m}$ excitation wavelength only targets the apical oxygen mode on sites with a chain oxygen vacancy (the YBCO 6 structure), and therefore the optical excitation is detuned from the phonon resonance in optimally doped YBCO 7 [43,47]. In Supplemental Material S4 [45], we also discuss the absence of any light induced coherence in underdoped YBCO after stimulation with $15 \mu \mathrm{m}$ pulses polarized parallel to the $\mathrm{CuO}_{2}$ planes (along the $a$ direction), resonant to an in-plane phonon mode.

In a superconductor at equilibrium, the superfluid density is proportional to the frequency-independent quantity $\left.\omega \sigma_{2}(\omega)\right|_{\omega \rightarrow 0}$. Along the $c$ axis, this response, approximated as $\omega \Delta \sigma_{2}(\omega)=\omega\left[\sigma_{2}\left(\omega, T<T_{c}\right)-\sigma_{2}\left(\omega, T>T_{c}\right)\right]$, measures the component of the superfluid that contributes to interlayer tunneling (proportional to $\omega_{p}^{2}$ ) and can be used to quantify the $c$ axis Josephson coupling strength [see Fig. 3(a.1)]. Here, we take $\omega \Delta \sigma_{2}(\omega, \tau)$ as a measure of light induced interbilayer coherence. This quantity, measured at the peak of the response, is plotted in Fig. 3(a.2) for YBCO 6.45 at different temperatures. Its mean value, $\left\langle\omega \Delta \sigma_{2}(\omega)\right\rangle$, was determined across all dopings by averaging $\omega \Delta \sigma_{2}(\omega)$ in the range where it remained frequency-independent $(\omega \lesssim 1.8 \mathrm{THz})$. The temperature dependence of $\left\langle\omega \Delta \sigma_{2}(\omega)\right\rangle$ is displayed in Fig. 3(b-e) for all compounds, along with the photo-susceptible volume fraction, $f$, extracted from the effective medium fits see Eq. (2). Due to the inhomogeneous nature of the excitation, the measured $\left\langle\omega \Delta \sigma_{2}(\omega)\right\rangle$ was rescaled by $f$ at all measured dopings. 

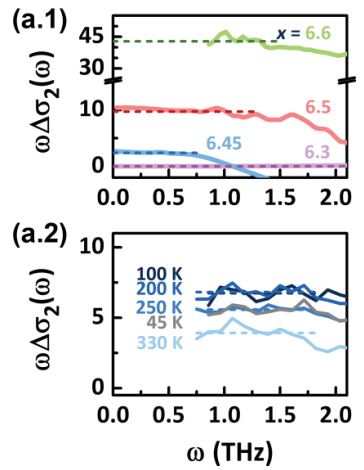
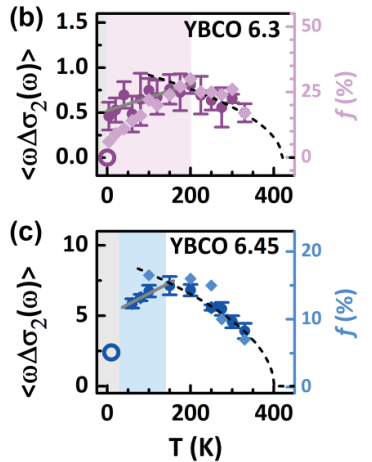
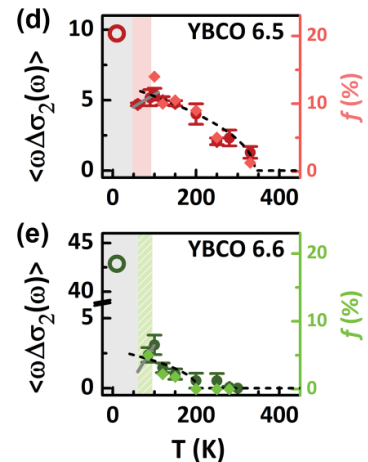

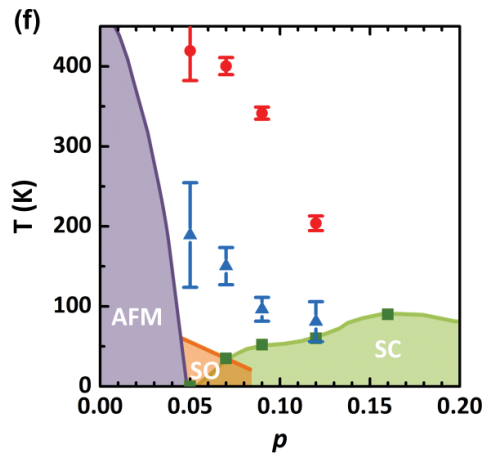

FIG. 3. Phase diagram of the transient state above $\boldsymbol{T}_{\boldsymbol{c}}$. (a.1) Equilibrium changes in $\omega \sigma_{2}(\omega)$ across the superconducting transition, expressed in units of THz $/(\Omega \cdot \mathrm{cm})$. (a.2) Light- induced $\omega \Delta \sigma_{2}(\omega, \tau=0.8 \mathrm{ps})$ measured in YBCO 4.5 at five different temperatures. Dashed lines indicate the mean values, $\left\langle\omega \Delta \sigma_{2}(\omega)\right\rangle$, extracted to quantify the strength of interbilayer coupling (see main text). (b-e) Frequency-averaged $\left\langle\omega \Delta \sigma_{2}(\omega)\right\rangle$, plotted as a function of temperature (filled circles). Equilibrium $\left\langle\omega \Delta \sigma_{2}(\omega)\right\rangle$ values measured at $5 \mathrm{~K}$ are also shown (empty circles). Diamonds refer to the photo-susceptible volume fractions, $f$, extracted from effective medium fits. The region $T<T^{\prime \prime}$ is indicated by a colored background for each doping. Linear fits to $\left\langle\omega \Delta \sigma_{2}(\omega)\right\rangle$ are displayed in this region as gray lines. At $T>T^{\prime \prime},\left\langle\omega \Delta \sigma_{2}(\omega)\right\rangle$ is well reproduced by a mean field behavior of the type $\propto \sqrt{1-T / T^{\prime}}$ (black dashed lines). (f) $T^{\prime}$ (red circles) and $T^{\prime \prime}$ (blue triangles) values, extracted from the fits, are plotted on the YBCO phase diagram. The equilibrium superconducting transition $T_{c}$, determined by dc magnetization, is denoted by green squares.

Two distinct temperature regimes are observed for $\left\langle\omega \Delta \sigma_{2}(\omega)\right\rangle$. Just above $T_{c}$, this quantity increases with temperature up to a crossover point $T^{\prime \prime}$. Above $T^{\prime \prime}$, the response follows the mean field behavior typical of a superfluid, $\left\langle\omega \Delta \sigma_{2}(\omega)\right\rangle \propto \sqrt{1-T / T^{\prime}}$, dropping to zero at a temperature $T^{\prime}$. The $T^{\prime \prime}$ crossover temperature is estimated by the intersection of a linear fit to the low-temperature regime $\left\langle\omega \Delta \sigma_{2}(\omega)\right\rangle$ (gray line) and the mean field fit to the high-temperature regime $\left\langle\omega \Delta \sigma_{2}(\omega)\right\rangle$ (black dashed line).
These two temperature scales are plotted on the phase diagram in Fig. 3(f). The similarity between $T^{\prime}$ and the $T^{*}$ line associated with the pseudogap phase is apparent. The $T^{\prime \prime}$ crossover appears to track the $T_{\mathrm{ELC}}$ scale, identified in Refs. [48,49] as the electronic liquid crystal (ELC) temperature, where there is an onset of nematic behavior due to collective excitations between spins. The reduction of $\left\langle\omega \Delta \sigma_{2}(\omega)\right\rangle$ below this temperature suggests that the ELC ground state may be competing with the light induced phase.

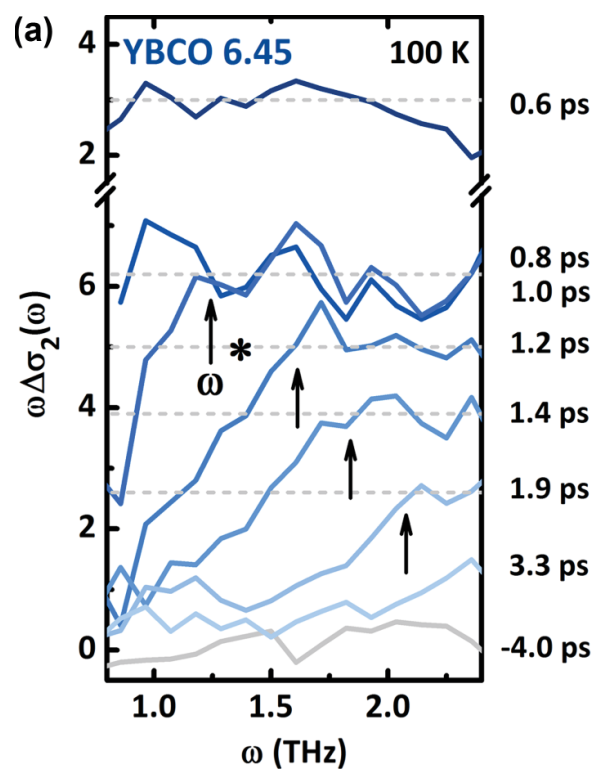

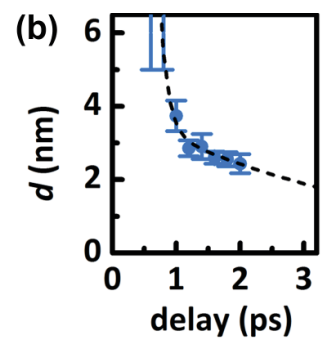
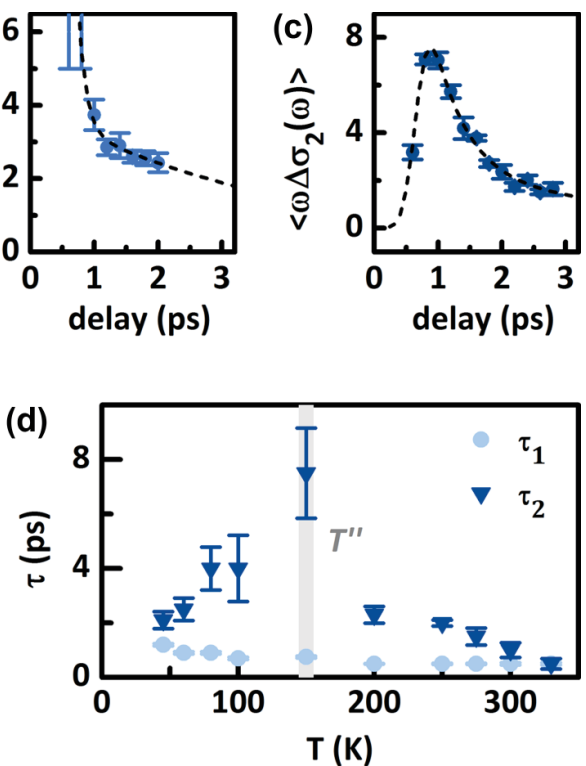

FIG. 4. Decoherence-driven relaxation of the transient state. (a) Transient $\omega \Delta \sigma_{2}(\omega)$ measured in YBCO 6.45 at $T=100 \mathrm{~K}$, for different pump-probe delays. A low-frequency downturn appears below a characteristic frequency $\omega^{*}$. (b) Relaxation of the coherence length $d=$ $2 \omega_{p} L / \omega^{*}$ (see main text) of the transient high-mobility state. A double exponential fit is displayed as a dashed line. (c) Corresponding relaxation of $\left\langle\omega \Delta \sigma_{2}(\omega)\right\rangle$, showing similar time constants. (d) Relaxation time constants of $\left\langle\omega \Delta \sigma_{2}(\omega)\right\rangle$, plotted as a function of temperature. The lifetime peaks near $T^{\prime \prime}$. 


\section{B. Relaxation of the light-induced state}

We now focus on the relaxation dynamics of the transient $c$ axis coupling, which is characterized by a drop in the imaginary conductivity below a frequency $\omega^{*}$, along with a decrease in the overall magnitude of $\omega \Delta \sigma_{2}(\omega)$. The time evolution of $\omega \Delta \sigma_{2}(\omega)$ after excitation is shown in Fig. 4(a) for YBCO 6.45 at $100 \mathrm{~K}$, while extended data sets are reported in Supplemental Material S5 [45]. The deviation from a frequency-independent behavior for $\omega \Delta \sigma_{2}(\omega)$ indicates an effective scattering rate $\omega^{*} \cong \Gamma$ in Eq. (1) for the interlayer coupling that increases in time, corresponding to a decrease in the coherence length along the $c$ axis. This is reminiscent of the $\omega \sigma_{2}(\omega)$ measured in $\mathrm{La}_{2-x} \mathrm{Sr}_{x} \mathrm{CuO}_{4}[9]$ and $\mathrm{Bi}_{2} \mathrm{Sr}_{2} \mathrm{CaCu}_{2} \mathrm{O}_{8+\delta}$ [11] near $T_{c}$, which was ascribed to fluctuations in the superconducting correlation length and time scale.

The coherence length $d$ of the transient state can be expressed as $d=2 \omega_{p} L / \omega^{*}$, where $L$ is the interbilayer spacing. This is plotted as a function of time delay in Fig. 4(b). The drop in coherence follows a double exponential fit, with fast $\left(\tau_{1} \lesssim 1 \mathrm{ps}\right)$ and slow $\left(\tau_{2} \simeq 4 \mathrm{ps}\right)$ relaxation components. The overall magnitude of $\left\langle\omega \Delta \sigma_{2}(\omega)\right\rangle$, measured for $\omega>\omega^{*}$, also follows a similar decay, as shown in Fig. 4(c). The temperature dependence of the $\left\langle\omega \Delta \sigma_{2}(\omega)\right\rangle$ relaxation timescales is displayed in Fig. 4(d). Remarkably, $\tau_{2}$ increases with increasing temperature until a turning point near $T^{\prime \prime} \approx$ $150 \mathrm{~K}$, and then it drops off, preserving a small but finite value even at the highest temperature measured.

During the relaxation, a second distinct phenomenon emerges. The light induced plasma resonance splits into two closely spaced modes. The splitting is most clearly visualized in the energy loss function (LF), $\operatorname{LF}(\omega)=-\operatorname{Im}[1 / \tilde{\varepsilon}(\omega)]$, displayed in Fig. 5 for YBCO 6.45 at $200 \mathrm{~K}$. At $\tau=0.8$ ps, the light induced plasma mode produces a single peak in the

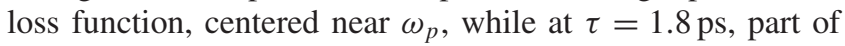
the spectral weight has blueshifted, resulting in two peaks separated by $\sim 1 \mathrm{THz}$. Correspondingly, $\sigma_{1}(\omega)$ develops a peak at an intermediate frequency.

Spatial variations of the plasma resonance frequency, for example, due to inhomogeneities, would simply result in a broadening of the plasmon [32]. The appearance of two distinct modes is instead indicative of the development of two inequivalent interbilayer couplings. A theoretical description of multiple, inequivalent junctions was developed in Refs. [32] and [33] to account for the bilayer structure of $\mathrm{YBa}_{2} \mathrm{Cu}_{3} \mathrm{O}_{6+\delta}$. We employ this same model for the split resonance observed during the relaxation dynamics, resulting in a complex dielectric function $\tilde{\varepsilon}_{2 p}$, described by the expression:

$$
\frac{\varepsilon_{\infty}}{\tilde{\varepsilon}_{2 p}}=\frac{\omega^{2} \tilde{z}_{1}}{\omega^{2}-\omega_{p 1}^{2}+i \omega \Gamma_{1}}+\frac{\omega^{2} \tilde{z}_{2}}{\omega^{2}-\omega_{p 2}^{2}+i \omega \Gamma_{2}} .
$$

Here, $\omega_{p 1}$ and $\omega_{p 2}$ are the two plasma frequencies, and $\Gamma_{1,2}$ captures the incoherent contributions to the response. The relative weights of each mode, $\tilde{z}_{1,2}$, are related to the geometry and electronic compressibility of each junction [33]. Effective medium fits, substituting $\tilde{\varepsilon}_{a}=\tilde{\varepsilon}_{2 p}$ in Eq. (2), are shown as dashed lines in Fig. 5. The two responses used in the effective medium are shown in Fig. 5(c). The peak in $\sigma_{1}(\omega)$ corresponds to a "transverse" plasma mode [33], centered at frequency $\omega_{T}=\sqrt{\tilde{z}_{1} \omega_{p 2}^{2}+\tilde{z}_{2} \omega_{p 1}^{2}}$. This splitting
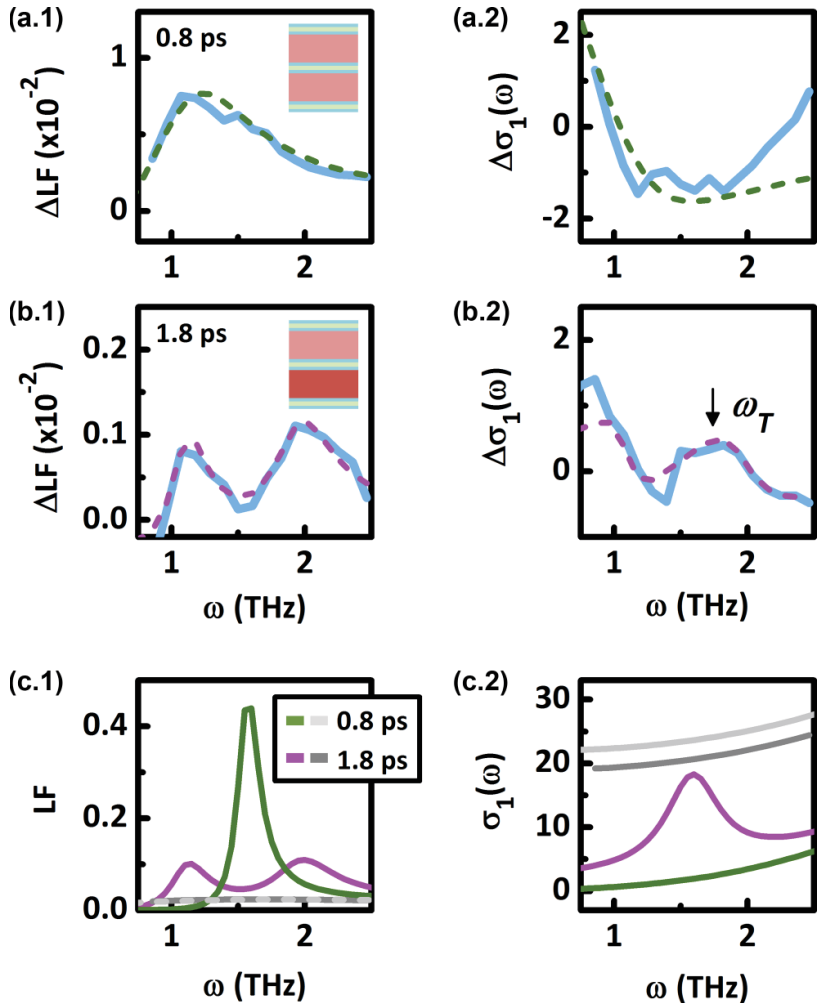

FIG. 5. Splitting of the plasma resonance during relaxation. (a-b) Pump-induced changes in the loss function and in the real part of the optical conductivity, measured in YBCO 6.45 at $T=200 \mathrm{~K}$ at two selected time delays. The splitting of the plasma mode (b.1) and the appearance of a peak in $\sigma_{1}(\omega)$ (b.2), observed at $\tau=1.8 \mathrm{ps,}$ indicate that two inequivalent interbilayer junctions have formed. Effective medium fits to the data (see main text) are shown as dashed lines. (c) Loss function and real conductivity of the two effective medium components, as extracted from the fits.

of the transient plasmon is a feature of the relaxation for all dopings, at all temperatures where the mode could be generated (see Supplemental Material S6 [45]).

A split Josephson plasmon could be caused by the generation of phase slips or vortices during relaxation, in a manner similar to the thermal vortex regime that forms an extended dome above $T_{c}$ in the equilibrium phase diagram of cuprates $[8,11,13,50]$. Vortices produced by an applied magnetic field along the $\mathrm{CuO}_{2}$ planes have been shown to induce a splitting of the equilibrium interbilayer Josephson plasmon in a similar fashion [34,35,51]. The spatial distribution of the phase variation determines the degree and shape of the splitting [36].

\section{SUMMARY}

The most natural implication of all results reported here involves transient light induced superconducting fluctuations at unprecedented high temperatures [40,41]. This possibility has been raised in previous papers, and the data reported here provide further support to this view, especially in relation to an alternative interpretation that posits the formation of a plasma of high-mobility quasiparticles. Note that such a quasiparticle plasma would have to be at the same frequency 
of the Josephson plasma edge below $T_{c}$, with the observed low scattering rates $(\Gamma \lesssim 0.5 \mathrm{THz}$ ), which are difficult to explain in terms of interlayer hopping of incoherent carriers.

Most importantly, the time evolution of the transient plasmon points to decoherence as an important driver of the relaxation to the ground state. This occurs through a collapse of the coherence length $d$ (see Fig. 4) or, equivalently, via an increase in the effective scattering rate $\Gamma$ see Eq. (1). In contrast, the response of Drude quasiparticles following photo-excitation in doped semiconductors typically results in a relaxation through a depletion of the carrier density at constant scattering time [52,53], or with a decrease in the scattering rate $[54,55]$. Finally, the observed splitting of the plasma mode, which fits nicely in a picture of a superfluid developing phase slips, cannot be explained by a plasma of mutually incoherent quasiparticles.

In summary, we have reported on an extensive study of the light induced coherent interlayer coupling in $\mathrm{YBa}_{2} \mathrm{Cu}_{3} \mathrm{O}_{6+\delta}$ throughout its pseudogap phase. Both the amplitude and lifetime of the response indicate the existence of a temperature scale $T^{\prime \prime}$ below which the transient plasmon is suppressed.
This temperature coincides with the onset of collective spin excitations, suggesting that spin ordering may depress light induced coherence. Furthermore, the relaxation of the light induced state appears to follow dynamics that are well aligned with the loss of phase coherence in the tunneling of a superfluid through the layers. This observation further supports an analogy with transient superconducting fluctuations. One challenge for future work is to extend the lifetime of the transient state, supporting the interlayer coupling against decoherence effects.

\section{ACKNOWLEDGMENTS}

The research leading to these results received funding from the European Research Council under the European Unions Seventh Framework Programme (FP7/2007-2013)/ERC Grant Agreement No. 319286 (QMAC). We acknowledge support from the Deutsche Forschungsgemeinschaft via the excellence cluster 'The Hamburg Centre for Ultrafast Imaging — Structure, Dynamics and Control of Matter at the Atomic Scale' and the priority program SFB925.
[1] T. Timusk and B. Statt, The pseudogap in high-temperature superconductors: An experimental survey, Rep. Prog. Phys. 62, 61 (1999).

[2] Z. A. Xu, N. P. Ong, Y. Wang, T. Kakeshita, and S. Uchida, Vortex-like excitations and the onset of superconducting phase fluctuation in underdoped $\mathrm{La}_{2-x} \mathrm{Sr}_{x} \mathrm{CuO}_{4}$, Nature 406, 486 (2000).

[3] D. N. Basov and T. Timusk, Electrodynamics of high- $T_{c}$ superconductors, Rev. Mod. Phys. 77, 721 (2005).

[4] M. Hücker, M. v. Zimmermann, G. D. Gu, Z. J. Xu, J. S. Wen, Guangyong Xu, H. J. Kang, A. Zheludev, and J. M. Tranquada, Stripe order in superconducting $\mathrm{LaBaCuO}(0.095 \leqslant$ $x \leqslant 0.155)$, Phys. Rev. B 83, 104506 (2011).

[5] A. Dubroka, M. Rössle, K. W. Kim, V. K. Malik, D. Munzar, D. N. Basov, A. A. Schafgans, S. J. Moon, C. T. Lin, D. Haug, V. Hinkov, B. Keimer, Th. Wolf, J. G. Storey, J. L. Tallon, and C. Bernhard, Evidence of a Precursor Superconducting Phase at Temperatures as High as $180 \mathrm{~K}$ in $R \mathrm{Ba}_{2} \mathrm{Cu}_{3} \mathrm{O}_{7-x}(\mathrm{R}=\mathrm{Y}, \mathrm{Gd}, \mathrm{Eu})$ Superconducting Crystals From Infrared Spectroscopy, Phys. Rev. Lett. 106, 047006 (2011).

[6] Q. Li, M. Hücker, G. D. Gu, A. M. Tsvelik, and J. M. Tranquada, Two-dimensional Superconducting Fluctuations in Stripe-ordered $\mathrm{La}_{1.875} \mathrm{Ba}_{0.125} \mathrm{CuO}_{4}$, Phys. Rev. Lett. 99, 067001 (2007).

[7] E. Uykur, K. Tanaka, T. Masui, S. Miyasaka, and S. Tajima, Persistence of the Superconducting Condensate Far Above the Critical Temperature of $\mathrm{YBa}_{2}(\mathrm{Cu}, \mathrm{Zn})_{3} \mathrm{O}_{y}$ Revealed by $c$-axis Optical Conductivity Measurements for Several Zn Concentrations and Carrier Doping Levels, Phys. Rev. Lett. 112, 127003 (2014).

[8] V. J. Emery and S. A. Kivelson, Importance of phase fluctuations in superconductors with small superfluid density, Nature 374, 434 (1995).

[9] L. S. Bilbro, R. Valdés Aguilar, G. Logvenov, O. Pelleg, I. Bozovic, and N. P. Armitage, Temporal correlations of super- conductivity above the transition temperature in $\mathrm{La}_{2-x} \mathrm{Sr}_{x} \mathrm{CuO}_{4}$ probed by terahertz spectroscopy, Nat. Phys. 7, 298 (2011).

[10] D. Nakamura, Y. Imai, A. Maeda, and I. Tsukada, Superconducting fluctuation investigated by $\mathrm{THz}$ conductivity of $\mathrm{La}_{2-x} \mathrm{Sr}_{x} \mathrm{CuO}_{4}$ thin films, J. Phys. Soc. Jpn. 81, 044709 (2012).

[11] J. Corson, R. Mallozzi, J. Orenstein, J. N. Eckstein, and I. Bozovic, Vanishing of phase coherence in underdoped $\mathrm{Bi}_{2} \mathrm{Sr}_{2} \mathrm{CaCu}_{2} \mathrm{O}_{8+\delta}$, Nature 398, 221 (1999).

[12] C. Stock, W. J. L. Buyers, R. Liang, D. Peets, Z. Tun, D. Bonn, W. N. Hardy, and R. J. Birgeneau, Dynamic stripes and resonance in the superconducting and normal phases of $\mathrm{YBa}_{2} \mathrm{Cu}_{3} \mathrm{O}_{6.5}$ ortho-II superconductor, Phys. Rev. B 69, 014502 (2004).

[13] Y. Wang, L. Li, and N. P. Ong, Nernst effect in high- $T_{c}$ superconductors, Phys. Rev. B 73, 024510 (2006).

[14] L. Li, Y. Wang, S. Komiya, S. Ono, Y. Ando, G. D. Gu, and N. P. Ong, Diamagnetism and Cooper pairing above $T_{c}$ in cuprates, Phys. Rev. B 81, 054510 (2010).

[15] J. M. Tranquada, B. J. Sternlieb, J. D. Axe, Y. Nakamura, and S. Uchida, Evidence for stripe correlations of spins and holes in copper oxide superconductors, Nature 375, 561 (1995).

[16] V. J. Emery, S. A. Kivelson, and J. M. Tranquada, Stripe phases in high-temperature superconductors, Proc. Nat. Acad. Sci. 96, 8814 (1999).

[17] M. Fujita, H. Goka, K. Yamada, and M. Matsuda, Competition Between Charge- and Spin-density-wave Order and Superconductivity in $\mathrm{La}_{1.875} \mathrm{Ba}_{0.125-x} \mathrm{Sr}_{x} \mathrm{CuO}_{4}$, Phys. Rev. Lett. 88, 167008 (2002).

[18] H. H. Klauss, W. Wagener, M. Hillberg, W. Kopmann, H. Walf, F. J. Litterst, M. Hücker, and B. Büchner, From Antiferromagnetic Order to Static Magnetic Stripes: The Phase Diagram of $(\mathrm{La}, \mathrm{Eu})_{2-x} \mathrm{Sr}_{x} \mathrm{CuO}_{4}$, Phys. Rev. Lett. 85, 4590 (2000).

[19] M. Hücker, G. D. Gu, J. M. Tranquada, M. von Zimmermann, H.-H. Klauss, N.J. Curro, M. Braden, and B. Büchner, Coupling of stripes to lattice distortions in cuprates and nickelates, Physica C 460, 170 (2007). 
[20] G. Ghiringelli, M. L. Tacon, M. Minola, S. Blanco-Canosa, C. Mazzoli, N. B. Brookes, G. M. De Luca, A. Frano, D. G. Hawthorn, F. He, T. Loew, M. Moretti Sala, D. C. Peets, M. Salluzzo, E. Schierle, R. Sutarto, G. A. Sawatzky, E. Weschke, B. Keimer, and L. Braicovich, Long-range incommensurate charge fluctuations in $(\mathrm{Y}, \mathrm{Nd}) \mathrm{Ba}_{2} \mathrm{Cu}_{3} \mathrm{O}_{6+x}$, Science 337, 821 (2012).

[21] E. H. da Silva Neto, P. Aynajian, A. Frano, R. Comin, E. Schierle, E. Weschke, A. Gyenis, J. Wen, J. Schneeloch, Z. Xu, S. Ono, G. $\mathrm{Gu}, \mathrm{M}$. Le Tacon, and A. Yazdani, Ubiquitous interplay between charge ordering and high-temperature superconductivity in cuprates, Science 343, 393 (2014).

[22] R. Comin, A. Frano, M. M. Yee, Y. Yoshida, H. Eisaki, E. Schierle, E. Weschke, R. Sutarto, F. He, A. Soumyanarayanan, Y. He, M. Le Tacon, I. S. Elfimov, J. E. Hoffman, G. A. Sawatzky, B. Keimer, and A. Damascelli, Charge order driven by Fermi-arc instability in $\mathrm{Bi}_{2} \mathrm{Sr}_{2-x} \mathrm{La}_{x} \mathrm{Cu}_{2} \mathrm{O}_{6+\delta}$, Science 343, 390 (2014).

[23] W. Tabis, Y. Li, M. L. Tacon, L. Braicovich, A. Kreyssig, M. Minola, G. Dellea, E. Weschke, M. J. Veit, M. Ramazanoglu, A. I. Goldman, T. Schmitt, G. Ghiringhelli, N. Barišić, M. K. Chan, C. J. Dorow, G. Yu, X. Zhao, B. Keimer, and M. Greven, Charge order and its connection with Fermi-liquid charge transport in a pristine high- $T_{c}$ cuprate, Nat. Comm. 5, 5875 (2014).

[24] S.-C. Zhang, Recent developments in the SO(5) theory of high $T_{c}$ superconductivity, J. Phys. Chem. Solids 59, 1774 (1998).

[25] A. Himeda, T. Kato, and M. Ogata, Stripe States with Spatially Oscillating $d$-wave Superconductivity in the Two-dimensional $t-t^{\prime}-J$ Model, Phys. Rev. Lett. 88, 117001 (2002).

[26] E. Berg, E. Fradkin, E.-A. Kim, S. A. Kivelson, V. Oganesyan, J. M. Tranquada, and S. C. Zhang, Dynamical Layer Decoupling in a Stripe-ordered High- $T_{c}$ Superconductor, Phys. Rev. Lett. 99, 127003 (2007).

[27] L. E. Hayward, D. G. Hawthorn, R. G. Melko, and S. Sachdev, Angular fluctuations of a multicomponent order describe the pseudogap of $\mathrm{YBa}_{2} \mathrm{Cu}_{3} \mathrm{O}_{6+x}$, Science 343, 1336 (2014).

[28] W. E. Lawrence and S. Doniach, in Proceedings of 12th International Confrence on Low Temperature Physics, Kyoto, 1970, edited by E. Kanda (Keigaku, Tokyo, 1971), p. 361.

[29] S. Doniach and M. Inui, Long-range Coulomb interactions and the onset of superconductivity in the high- $T_{c}$ materials, Phys. Rev. B 41, 6668 (1990).

[30] M. Tachiki, T. Koyama, and S. Takahashi, Electromagnetic phenomena related to a low-frequency plasma in cuprate superconductors, Phys. Rev. B 50, 7065 (1994).

[31] L. N. Bulaevskii, M. Zamora, D. Baeriswyl, H. Beck, and J. R. Clem, Time-dependent equations for the phase differences and a collective mode in Josephson-coupled layered superconductors, Phys. Rev. B 50, 12831 (1994).

[32] D. van der Marel and A. A. Tsvetkov, Transverse optical plasmons in layered superconductors, Czech. J. Phys. 46, 3165 (1996).

[33] D. van der Marel and A. A. Tsvetkov, Transverse-optical Josephson plasmons: Equations of motion, Phys. Rev. B. 64, 024530 (2001).

[34] A. Pimenov, A. Loidl, D. Dulić, D. van der Marel, I. M. Sutjahja, and A. A. Menovsky, Magnetic Field Dependence of the Transverse Plasmon in $\mathrm{SmLa}_{0.8} \mathrm{Sr}_{0.2} \mathrm{CuO}_{4-\delta}$, Phys. Rev. Lett. 87, 177003 (2001).

[35] A. D. LaForge, W. J. Padilla, K. S. Burch, Z. Q. Li, S. V. Dordevic, K. Segawa, Y. Ando, and D. N. Basov, Interlayer elec- trodynamics and unconventional vortex state in $\mathrm{YBa}_{2} \mathrm{Cu}_{3} \mathrm{O}_{y}$, Phys. Rev. B 76, 054524 (2007).

[36] A. E. Koshelev, Electrodynamics of the Josephson vortex lattice in high temperature superconductors, Phys. Rev. B 76, 054525 (2007).

[37] D. Fausti, R. I. Tobey, N. Dean, S. Kaiser, A. Dienst, M. C. Hoffmann, S. Pyon, T. Takayama, H. Takagi, and A. Cavalleri, Light-induced superconductivity in a stripe-ordered cuprate, Science 331, 189 (2011).

[38] C. R. Hunt, D. Nicoletti, S. Kaiser, T. Takayama, H. Takagi, and A. Cavalleri, Two distinct kinetic regimes for the relaxation of light-induced superconductivity in $\mathrm{La}_{1.625} \mathrm{Eu}_{0.2} \mathrm{Sr}_{0.125} \mathrm{CuO}_{4}$, Phys. Rev. B 91, 020505(R) (2015).

[39] M. Först, R. I. Tobey, H. Bromberger, S. B. Wilkins, V. Khanna, A. D. Caviglia, Y.-D. Chuang, W. S. Lee, W. F. Schlotter, J. J. Turner, M. P. Minitti, O. Krupin, Z. J. Xu, J. S. Wen, G. D. Gu, S. S. Dhesi, A. Cavalleri, and J. P. Hill, Melting of Charge Stripes in Vibrationally Driven $\mathrm{La}_{1.875} \mathrm{Ba}_{0.125} \mathrm{CuO}_{4}$ : Assessing the Respective Roles of Electronic and Lattice Order in Frustrated Superconductors, Phys. Rev. Lett. 112, 157002 (2014).

[40] S. Kaiser, C. R. Hunt, D. Nicoletti, W. Hu, I. Gierz, H. Y. Liu, M. Le Tacon, T. Loew, D. Haug, B. Keimer, and A. Cavalleri, Optically induced coherent transport far above $T_{c}$ in underdoped $\mathrm{YBa}_{2} \mathrm{Cu}_{3} \mathrm{O}_{6+\delta}$, Phys. Rev. B 89, 184516 (2014).

[41] W. Hu, S. Kaiser, D. Nicoletti, C. R. Hunt, I. Gierz, M. C. Hoffmann, M. Le Tacon, T. Loew, B. Keimer, and A. Cavalleri, Optically enhanced coherent transport in $\mathrm{YBa}_{2} \mathrm{Cu}_{3} \mathrm{O}_{6.5}$ by ultrafast redistribution of interlayer coupling, Nat. Mater. 13, 705 (2014).

[42] M. Först, A. Frano, S. Kaiser, R. Mankowsky, C. R. Hunt, J. J. Turner, G. L. Dakovski, M. P. Minitti, J. Robinson, T. Loew, M. Le Tacon, B. Keimer, J. P. Hill, A. Cavalleri, and S. S. Dhesi, Femtosecond $x$ rays link melting of chargedensity wave correlations and light-enhanced coherent transport in $\mathrm{YBa}_{2} \mathrm{Cu}_{3} \mathrm{O}_{6.6}$, Phys. Rev. B 90, 184514 (2014).

[43] R. Mankowsky, A. Subedi, M. Först, S. O. Mariager, M. Chollet, H. T. Lemke, J. S. Robinson, J. M. Glownia, M. P. Minitti, A. Frano, M. Fechner, N. A. Spaldin, T. Loew, B. Keimer, A. Georges, and A. Cavalleri, Nonlinear lattice dynamics as a basis for enhanced superconductivity in $\mathrm{YBa}_{2} \mathrm{Cu}_{3} \mathrm{O}_{6.5}$, Nature 516, 71 (2014).

[44] R. Höppner, B. Zhu, T. Rexin, A. Cavalleri, and L. Mathey, Redistribution of phase fluctuations in a periodically driven cuprate superconductor, Phys. Rev. B 91, 104507 (2015).

[45] See Supplemental Material at http://link.aps.org/supplemental/ 10.1103/PhysRevB.94.224303 for additional data sets and details of the analysis.

[46] Quasiparticle contributions are accounted for in the effective medium fits by adding a small additional Drude term to the $1-f$ volume fraction. Within the THz frequency window, this is equivalent to adding a constant contribution to $\sigma_{1}(\omega)$.

[47] C. C. Homes, T. Timusk, D. A. Bonn, R. Liang, and W. N. Hardy, Optical properties along the $c$-axis of $\mathrm{YBa}_{2} \mathrm{Cu}_{3} \mathrm{O}_{6+x}$, for $x=0.5 \rightarrow 0.95$ : Evolution of the pseudogap, Physica C 254, 265 (1995).

[48] D. Haug, V. Hinkov, Y. Sidis, P. Bourges, N. B. Christensen, A. Ivanov, T. Keller, C. T. Lin, and B. Keimer, Neutron scattering study of the magnetic phase diagram of underdoped $\mathrm{YBa}_{2} \mathrm{Cu}_{3} \mathrm{O}_{6+x}$, New J. Phys. 12, 105006 (2010). 
[49] V. Hinkov, D. Haug, B. Fauqué, P. Bourges, Y. Sidis, A. Ivanov, C. Bernhard, C. T. Lin, and B. Keimer, Electronic liquid crystal state in the high-temperature superconductor $\mathrm{YBa}_{2} \mathrm{Cu}_{3} \mathrm{O}_{6.45}$, Science 319, 597 (2008).

[50] P. M. C. Rourke, I. Mouzopoulou, X. Xu, C. Panagopoulos, Y. Wang, B. Vignolle, C. Proust, E. V. Kurganova, U. Zeitler, Y. Tanabe, T. Adachi, Y. Koike, and N. E. Hussey, Phasefluctuating superconductivity in overdoped $\mathrm{La}_{2-x} \mathrm{Sr}_{x} \mathrm{CuO}_{4}$, Nat. Phys. 7, 455 (2011).

[51] K. M. Kojima, S. Uchida, Y. Fudamoto, and S. Tajima, New Josephson Plasma Modes in Underdoped $\mathrm{YBa}_{2} \mathrm{Cu}_{3} \mathrm{O}_{6.6}$ Induced by a Parallel Magnetic Field, Phys. Rev. Lett. 89, 247001 (2002).
[52] H. Němec, L. Fekete, F. Kadlec, P. Kŭzel, M. Martin, J. Mangeney, J. C. Delagnes, and P. Mounaix, Ultrafast carrier dynamics in $\mathrm{Br}^{+}$-bombarded InP studied by time-resolved terahertz spectroscopy, Phys. Rev. B 78, 235206 (2008).

[53] J. C. Delagnes, P. Mounaix, H. Němec, L. Fekete, F. Kadlec, P Kužel, M. Martin, and J. Mangeney, High photocarrier mobility in ultrafast ion-irradiated $\operatorname{In}_{0.53} \mathrm{Ga}_{0.47}$ As for terahertz applications, J. Phys. D: Appl. Phys. 42, 195103 (2009).

[54] J. Zielbauer and M. Wegener, Ultrafast optical pump THz-probe spectroscopy on silicon, Appl. Phys. Lett. 68, 1223 (1996).

[55] M. C. Beard, G. M. Turner, and C. A. Schmuttenmaer, Transient photoconductivity in GaAs as measured by time-resolved terahertz spectroscopy, Phys. Rev. B 62, 15764 (2000). 